\title{
Characterization of $\mathrm{Ni} / \mathrm{SnPb}$-TiW/Pt Flip Chip Interconnections in Silicon Pixel Detector Modules
}

\author{
A Karadzhinova ${ }^{1}$, A Nolvi ${ }^{2}$, J Härkönen ${ }^{1}$, P Luukka ${ }^{1}$, T Mäenpää ${ }^{1}$, E Tuominen ${ }^{1}$, \\ E Hæggström ${ }^{2}$, J Kalliopuska ${ }^{3}$, S Vähänen ${ }^{3}$ and I Kassamakov ${ }^{1,2}$
}

${ }^{1}$ Detector Laboratory, Helsinki Institute of Physics, PO Box 64, FI-00014, Helsinki, Finland

${ }^{2}$ Department of Physics, University of Helsinki, PO Box 64, FI-00014, Helsinki, Finland

${ }^{3}$ Advacam Ltd, Tietotie 3, Espoo, Finland

E-mail: aneliya.karadzhinova@ helsinki.fi

In contemporary high energy physics experiments, silicon detectors are essential for recording the trajectory of new particles generated by multiple simultaneous collisions. Modern particle tracking systems may feature 100 million channels, or pixels, which need to be individually connected to read-out chains. Silicon pixel detectors are typically connected to readout chips by flip-chip bonding using solder bumps.

High-quality electro-mechanical flip-chip interconnects minimizes the number of dead read-out channels in the particle tracking system. Furthermore, the detector modules must endure handling during installation and withstand heat generation and cooling during operation.

Silicon pixel detector modules were constructed by flip-chip bonding 16 readout chips to a single sensor. Eutectic $\mathrm{SnPb}$ solder bumps were deposited on the readout chips and the sensor chips were coated with TiW/Pt thin film UBM (under bump metallization). The modules were assembled at Advacam Ltd, Finland.

We studied the uniformity of the solder bumps using Scanning White Light Interferometry (SWLI). According to our results, the $\mathrm{Ni} / \mathrm{SnPb}-\mathrm{TiW} / \mathrm{Pt}$ interconnections fulfill the requirements in [1] for flip-chip bonding pixel detector modules.

This study proposes a way to decrease the number of dead channels of the silicon pixel detector modules by precisely measuring the soldered bump diameter to ensure that they fulfil the specifications.

Technology and Instrumentation in Particle Physics 2014

2-6 June, 2014

Amsterdam, the Netherlands 


\section{Introduction}

Silicon pixel detector modules comprise a 2D diode array bonded to an electronic chip [2]. Current sensing elements are silicon diodes. The readout chip (ROC) contains circuitry needed to read and process the signals collected by the diodes. A simple way to electrically connect the two chips is to mount them on a laminated substrate and use conventional wire bonding in a Chip-on-Board approach. This solution limits the number of channels to a few thousand.

The quality of the silicon pixel detector modules depend on the quality of the ROC, the sensor chip, and the connections between them. The connection can be done by wire bonding or flip-chip technology [3]. Flip-chip bonding (FCB) technology, known since the 1960s, has advanced due to commercial interest in high-density packaging. Flip-chip bonding has demonstrated better electrical performance ${ }^{1}$ and reliability than conventional wire bonding [3]. Lately FCB has advanced the fabrication of pixel systems, with narrow pitch $(55 \mu \mathrm{m})$ and many bumps. These two features make the flip-chip interconnection preferred in silicon pixel detector modules. In FCB one of the two chips is flipped, aligned, and then bonded to the other chip using solder bumps, conductive adhesives, or other conductive media. Although flip chip processing using electroplated solder bumps may seem to be a simpler technology than wire bonding, it faces challenges, mostly because the yield of uniform flip chip joints in imaging detectors or tracking applications has to be high (> 99.9\%).

Modern pixel detectors may feature 100 million sensing elements which need to be individually connected to their read-out chains. The high-quality of the soldered bumps and there reliable connectivity is therefore key for the success of pixel detectors and the most important step in the bonding process. For instance, the bumps have to be uniform in height to avoid open joints or solder bridges between adjacent pixels.

The Helsinki Institute of Physics (Finland) has several commitments at the European Organization for Nuclear Research, CERN. Together with company Advacam Inc. 250 silicon pixel detector modules will be bump-bonded and characterized for the upgrade of the inner Tracker of Compact Muon Solenoid experiment. Successful, trustworthy, timely, and economical manufacture of these modules require reliable and accessible quality assurance methods.

Imaging spherical objects is challenging, because their curvature causes the reflected light to be lost. Therefore one cannot see part of the sphere due to its steep slope. We observe a shadow and we can determine the diameter of the spheres by measuring the diameter of the sphere's shadow. By increasing the system magnification the imaged area of the sphere increases, at the expense of the field of view (fewer bonds at a time, which equals lower inspection rate).

1

As chip voltages drop and current requirements increase, it is advantageous to distribute power and ground directly to the core of the device. This can be done using large-area array solder bumps to minimize the voltage drop between the chips. This approach also minimizes simultaneous switching noise due to the voltage drop in the chip power distribution. On sensitive signal paths, additional power and ground bumps can be used to surround the sensitive I/O bumps.[4] 


\section{Methods}

\subsection{SWLI}

A Scanning White Light Interferometer (SWLI), Fig.1, can characterize surface topography. This full-field-of-view non-contact device measures the topography of surfaces. Based on the reflection from the measured surface of a white light beam into an interferometric objective, this technique provides quantitative information across large areas with $\pm 5 \mathrm{~nm}$ vertical resolution [5].

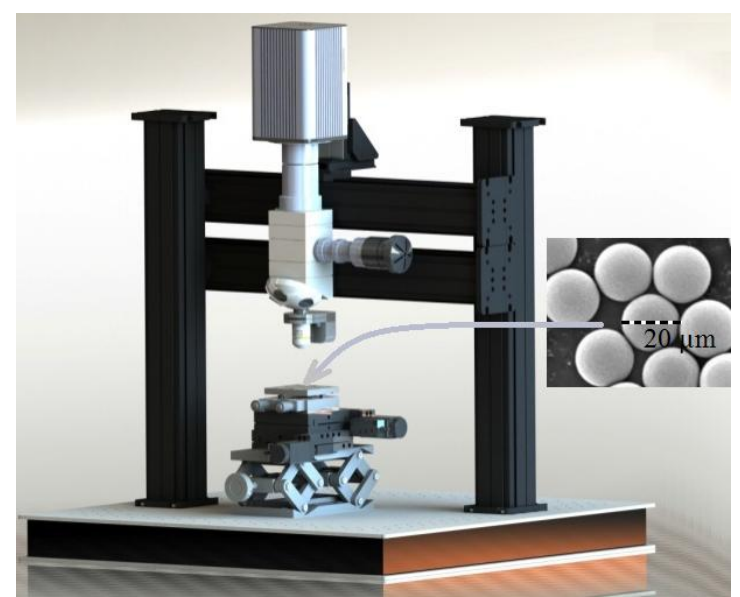

Fig. 1. Example of NIST calibrated spheres [6] examined with our Scanning white light interferometer.

\subsection{NIST certified $\mathrm{SiO}_{2}$ spheres}

We used $\mathrm{SiO}_{2}$ spheres, to determine the uncertainty of the SWLI set-up for characterizing spherical surfaces. Produced by Corpuscular Inc. [6] the spheres featured a National Institute of Standards and Technology (NIST) certified diameter of $20 \pm 1.2 \mu \mathrm{m}$ [7].

We pipetted a small amount of $1.0 \%$ solids aqueous dispersion containing the spheres onto glass microscope slides (Thermo Scientific, Menzel-Gläser, ca./env. 76 x $26 \mathrm{~mm}^{2}$ and $3.1 \mathrm{~nm}$ surface roughness) and left them to dry for an hour in $\mathrm{T}=22{ }^{\circ} \mathrm{C}$, and $\mathrm{R}_{\mathrm{H}}=25 \%$. Then the seeded slides were measured with the SWLI set-up. Five randomly chosen spheres were selected for further studies. Each sphere was measured three times (without moving the sample between the measurements).

\subsection{Bump-bonds}

Figure 2 shows the principle of the bump bonding technique. The studied bumps were deposited at Advacam Ltd. Eutectic SnPb solder bumps were deposited, Fig.3, on the readout wafers. The sensor wafers carried a TiW/Pt thin film UBM.

Nine neighbouring bump-bonds, Fig. 4, were randomly chosen for further studies. Each bump was measured with SWLI three times along four different directions (without sample replacement). We determined the measurement uncertainty of the single bond height based on the average value of these measurements (see the Result section).Mountain maps [9] software was used for 3D image reconstruction. 


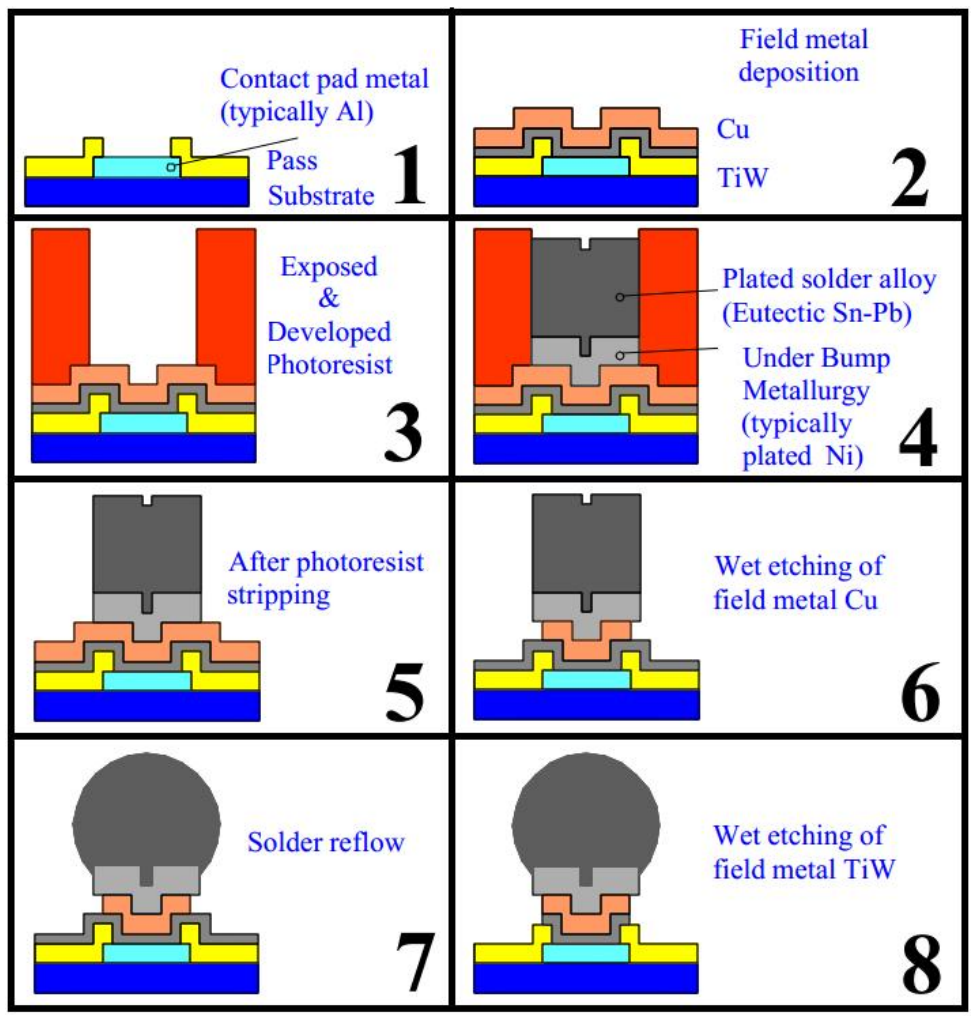

Fig. 2. Bump bonding technique [8].

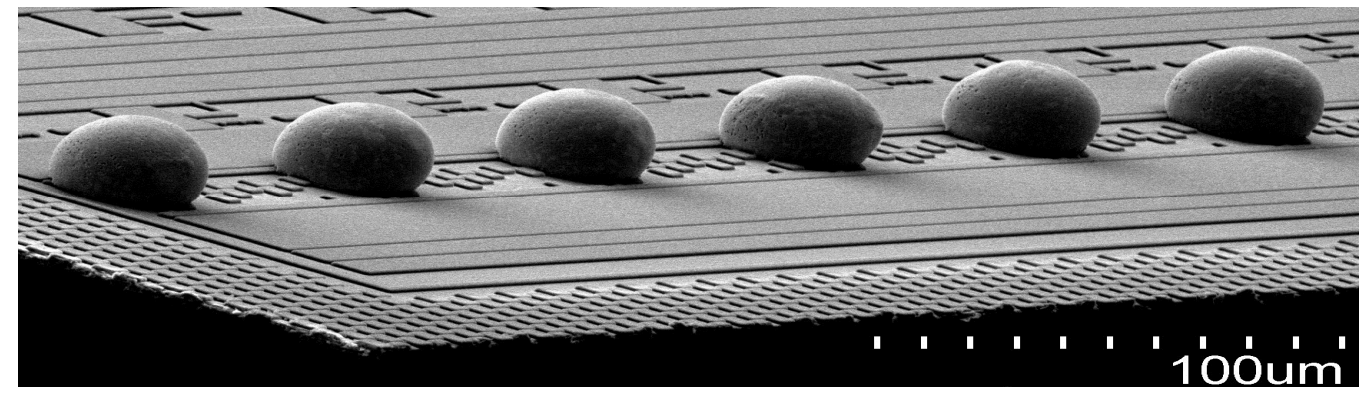

Fig. 3. Solder bumps imaged with scanning electron microscopy.

\section{Results}

\subsection{Spheres}

The average maximum visible height $\mathrm{H}$ of the spheres (see Fig.5 for a single sphere), was calculated based on four profiles (separated by $45^{\circ}$, see Fig. 4) across the visible summit of the profile of one sphere.

Based on the average value of the spheres' maximum height $\mathrm{H}$ measured with the interferometer set up, the calibration constant of the SWLI was $+0.40 \mu \mathrm{m}$ in the centre of the field of view and $+0.41 \mu \mathrm{m}$ at the edges of the field of view. This holds true within the calibration range stated above. The uncertainty in maximum bond height $\mathrm{H}$ estimate was calculated according to UKAS M3003 [10]. A coverage factor $\mathrm{k}=2$, provides a 95\% confidence level which in this particular case was $\pm 0.75 \mu \mathrm{m}$ in the centre of field of view and $\pm 0.70 \mu \mathrm{m}$ at 
the edges of the field of view. This number is larger than the $\pm 0.12 \mu \mathrm{m}$ measured for the NIST spheres since it also includes the nonuniformity of the bump bonds.

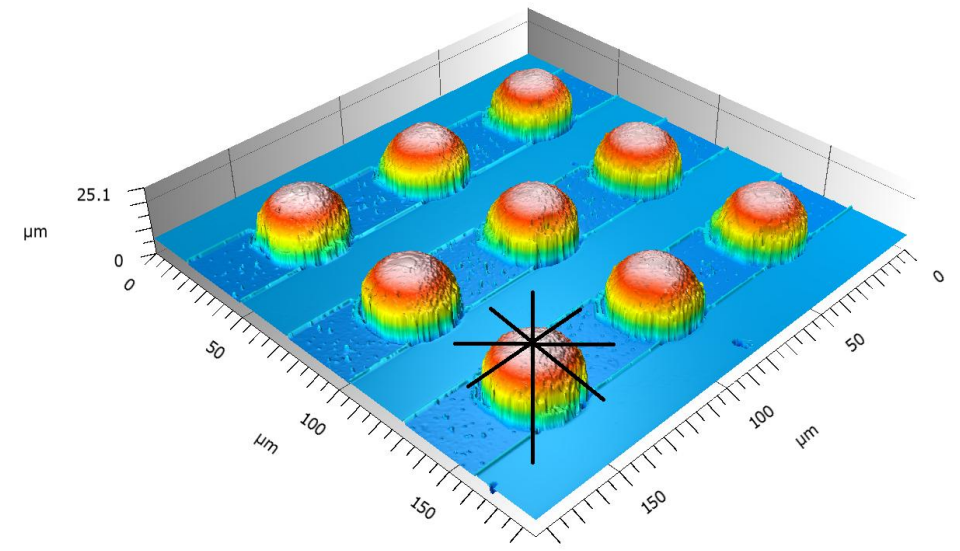

Fig. 4. 3D reconstruction of the nine neighbouring bumps imaged with SWLI. Indicated is also the polar grid showing the profile extraction.

The blue line of Fig. 5 shows what we see with the SWLI - the top and the sphere shadow on the base surface. The orange dash line shows the invisible for the SWLI sphere profile.

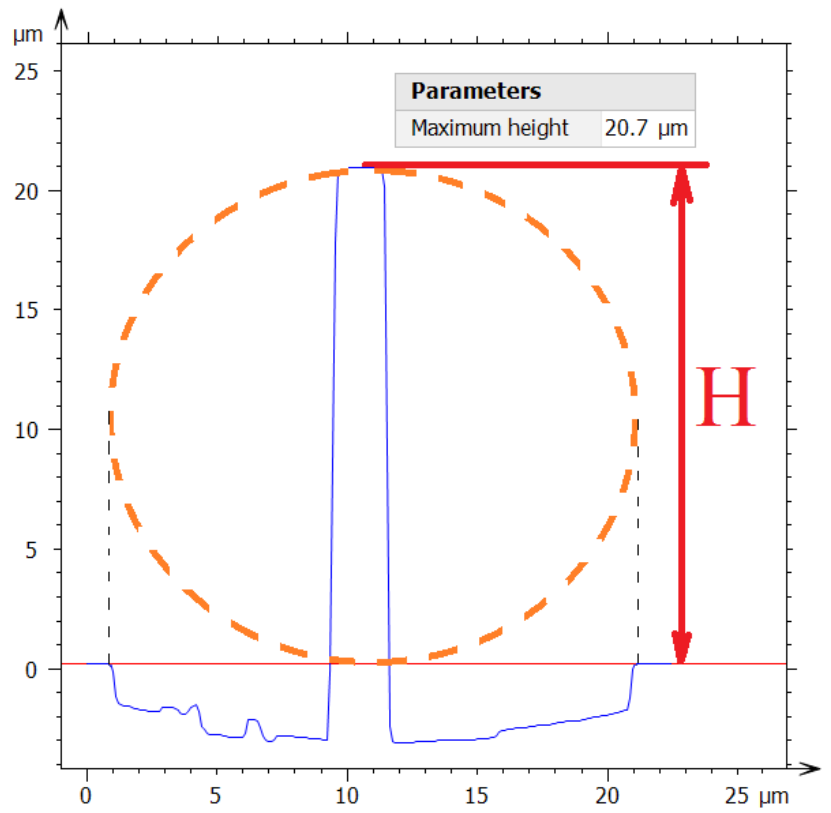

Fig. 5. Maximum height $H$ measured from the top of a single $\mathrm{SiO}_{2}$ sphere to the microscope slide. The contour of the half sphere body should follow the orange dashed line.

\subsection{Bump-bonds}

Figure 7 shows the average maximum height (Fig. 8 shows the four profiles of a soldered bump and Table 1 contain the maximum height data for it) for the nine bumps after applying the SWLI measurement correction of $+0.98 \mu \mathrm{m}$ corresponding to the SLWI pixel size obtained during the NIST sphere measurements, as required by UKAS M3003 [10]. The bump-bonds maximum height was determined to be $17.63-18.76 \mu \mathrm{m}$ (range), which falls inside the $\pm 1 \mu \mathrm{m}$ tolerance, typically archived during UBM processing 


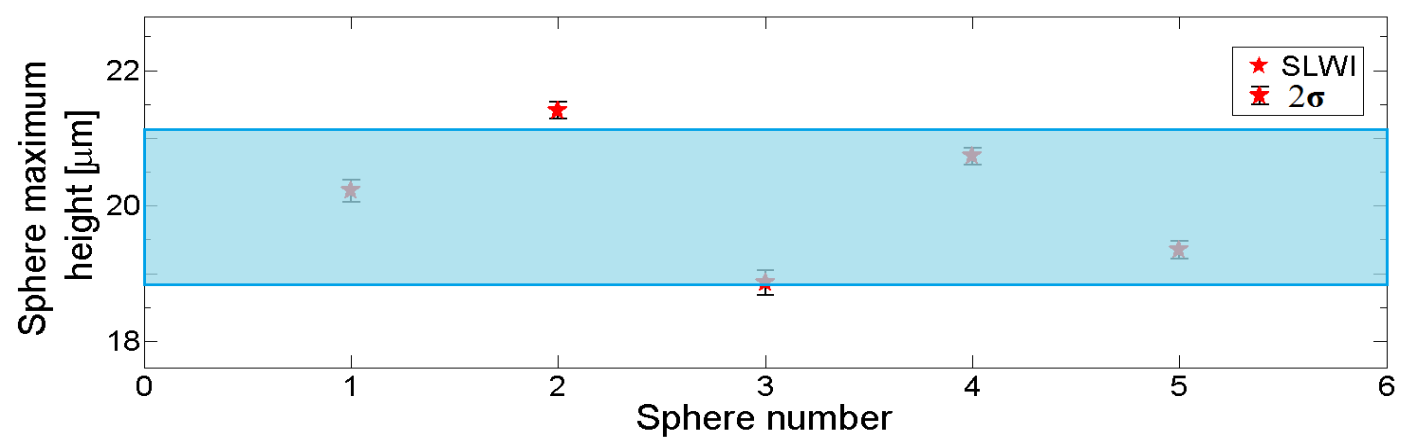

Fig. 6. Maximum height $H$ of the five $\mathrm{SiO}_{2}$ spheres and the NIST specifications in blue.

\subsection{Bump-bonds}

Figure 7 shows the average maximum height (Fig. 8 shows the four profiles of a soldered bump and Table 1 contain the maximum height data for it) for the nine bumps after applying the SWLI measurement correction of $+0.98 \mu \mathrm{m}$ corresponding to the SLWI pixel size obtained during the NIST sphere measurements, as required by UKAS M3003 [10]. The bump-bonds maximum height was determined to be $17.63-18.76 \mu \mathrm{m}$ (range), which falls inside the $\pm 1 \mu \mathrm{m}$ tolerance, typically archived during UBM processing.

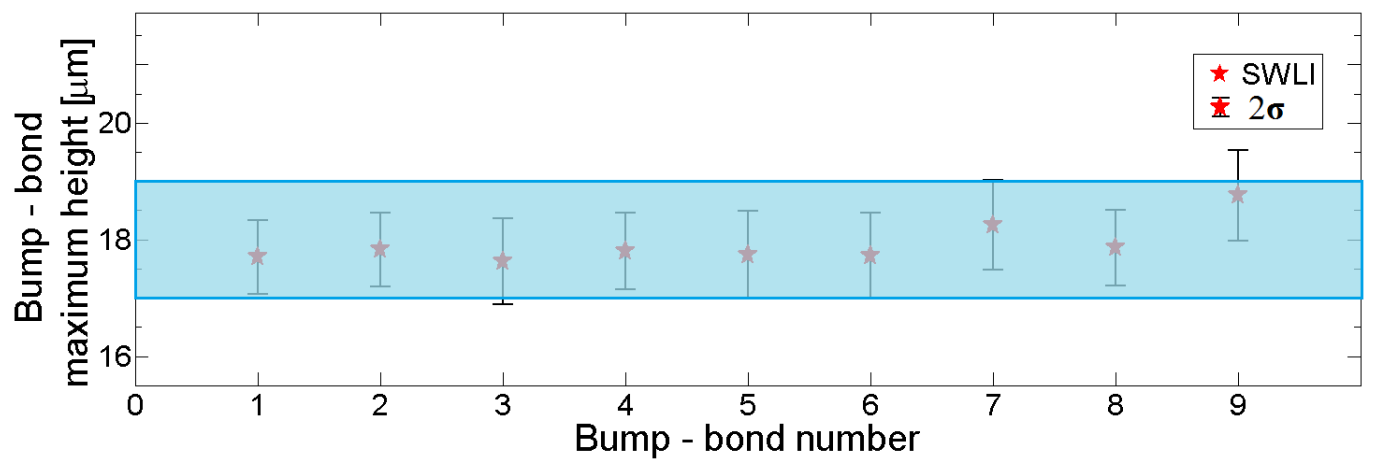

Fig. 7. Measured maximum height $H$ of the 9 bumps. The typical UBM specification $( \pm 1 \mu \mathrm{m})$ boundaries are indicated in blue.

Table 1 Maximum height measurement of bump bond \#5

\begin{tabular}{ccc}
\hline Feature & Before calibration & After calibration \\
\hline $0^{\circ}$ & $18.03 \pm 0.31 \mu \mathrm{m}$ & $17.63 \pm 0.31 \mu \mathrm{m}$ \\
$45^{\circ}$ & $18.60 \pm 0.30 \mu \mathrm{m}$ & $18.19 \pm 0.31 \mu \mathrm{m}$ \\
$90^{\circ}$ & $18.03 \pm 0.31 \mu \mathrm{m}$ & $17.63 \pm 0.31 \mu \mathrm{m}$ \\
$135^{\circ}$ & $17.90 \pm 027 \mu \mathrm{m}$ & $17.50 \pm 0.27 \mu \mathrm{m}$ \\
\hline
\end{tabular}

\section{Discussion}

The SWLI height measurement was calibrated by measuring spheres with known diameter (it is important that they are in close contact to the microscope slide surface and that they are not covered by extra films of moisture etc.). This calibration is valid for heights within the height range $18.0-22 \mu \mathrm{m}$. This calibration approach was used to assure the vertical resolution of our SWLI instrument and to prove its ability to scan spherical objects. 

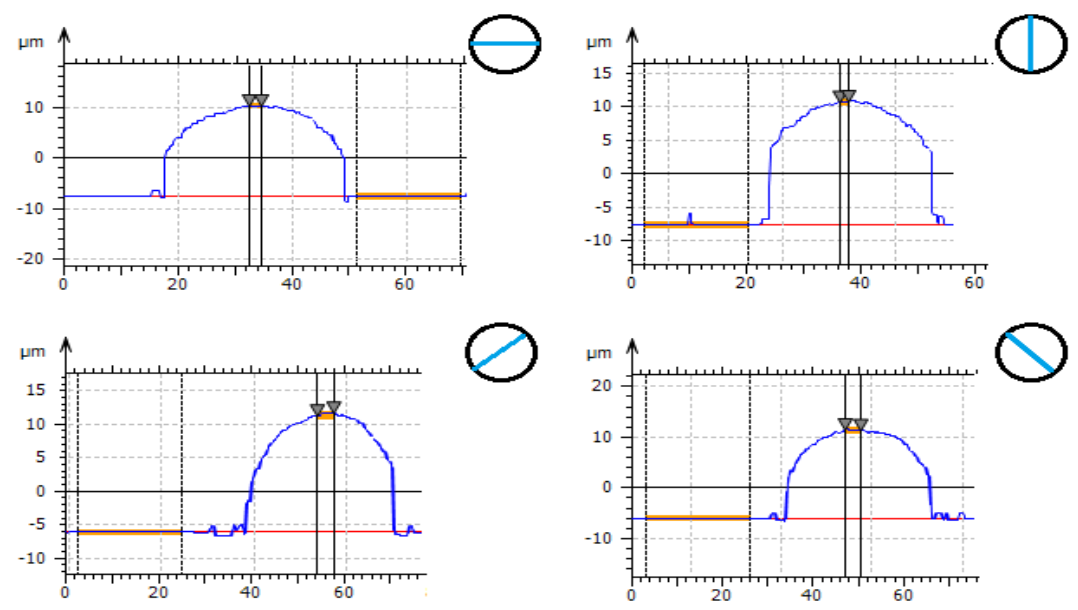

Fig. 8. The four profiles of bump bond \#5.

\section{Conclusion}

The SWLI instrument was calibrated using NIST $\mathrm{SiO}_{2}$ spheres. The bump-bond heights were $17.63 \mu \mathrm{m}-18.76 \mu \mathrm{m}$ (range), which falls inside the $\pm 1 \mu \mathrm{m}$ tolerance. All calculations and their corrections were done according to the UKAS M3003 requirement.

This study proposes a way to decrease the number of dead channels in silicon pixel detector modules by precisely measuring the soldered bump diameter.

\section{References}

[1] Satu Savolainen-Pulli, Jaakko Salonen, Jorma Salmi, Sami Vähänen, Experiences in flip chip production of radiation detectors, Nuclear Instruments and Methods in Physics Research A 565 (2006) 314-319

[2] R. Baur, W. Bertl, The CMS pixel vertex detector, Nuclear Physics B - Proceedings Supplements, 78, p. 293, 1999.

[3] J. H. Lau, Flip Chip Technologies, McGraw - Hill, New York, pp. 565, 1996.

[4] P. Elenius and L. Levine, Comparing flip-chip and wire-bond interconnection technologies, Chip scale review (July 2000) 81-7, http://www.processsolutionsconsulting.com/pdf/Flip_Bump/csr-700.pdf, June 2014 (Online).

[5] Jeremias Seppä; Ivan Kassamakov; Ville Heikkinen; Anton Nolvi; Tor Paulin; Antti Lassila; Edward Hæggström, Quasidynamic calibration of stroboscopic scanning white light interferometer with a transfer standard, Optical Engineering 52(12), December 2013

[6] Corpuscular Inc., 3590 Route 9, Suite 107 Cold Spring, New York 10516, http://www.microspheres-nanospheres.com/, June 2014 (Online).

[7] National Institute of Standards and Technology, 100 Bureau Dr, Gaithersburg, MD 20899, USA, http://www.nist.gov/, 2014 (Online).

[8] J. Salmi and J. Salonen, "Solder Bump Flip Chip Bonding for Pixel Detector Hybridization," Workshop on Bonding and Die Attach Technologies, CERN, Geneva, Switzerland, June 11-12, 2003.

[9] Digital Surf, 25000 Besançon, France, http://www.digitalsurf.fr/en/index.html , June 2014 (Online). 
[10] M3003-The Expression of Uncertainty and Confidence in Measurement, UKAS, 3rd, United Kingdom Accreditation Service, Feltham, Middlesex, The United Kingdom, (2012) 